\title{
Verlängerte biologische Halbwertszeit der sulfatierten Mukopolysaccharide bei Alloxandiabetes als Ursache der diabetischen Mesenchymstoffwechselstörung
}

\author{
Von W. H. Hauss, G. Junge-Hülsing, I. Kuckulies, H. Otto, J. Rawytsch und H. WagneR \\ Aus der Medizinischen Klinike und Poliklinik der Universität Münster \\ (Direktor Prof. Dr. W. H. Hauss)
}

(Eingegangen am 3. April 1968)

\section{Herrn Prof. Dr. Dr. Ernst Schütte zum 60. Geburtstag in freundschaftlicher Verbundenbeit gewidmet}

\begin{abstract}
Bei alloxandiabetischen Ratten und gesunden Kontrolltieren wurden Synthese- und Umsatzraten der sulfatierten Mukopolysaccharide mit Hilfe von ${ }^{35}$ S-Sulfat gemessen.

Bei den alloxandiabetischen Tieren fand sich in Ubereinstimmung mit früheren Befunden eine Verminderung der Syntheserate der sulfatierten Mukopolysaccharide auf etwa die Hälfte der bei den unbehandelten Kontrolltieren gefundenen Werte. Die zusätzlich durchgeführten Bestimmungen der Umsatzraten der sulfatierten Mukopolysaccharide in den verschiedenen Organen ergaben bei den alloxandiabetischen Tieren eine Verlängerung der biologischen Halbwertzeiten auf mehr als das Dreifache der bei Kontrolltieren gefundenen Werte. Das Ausmaß der Verlängerung der Halbwertszeiten der sulfatierten Mukopolysaccharide übertraf also bei den alloxandiabetischen Tieren das Ausmaß der Synthesehemmung.
\end{abstract}

$\mathrm{Da}$ die beschriebenen Veränderungen des SMPS1)-Umsatzes wahrscheinlich eine direkte Folge des Insulinmangels bei Alloxandiabetes sind, ist es wahrscheinlich, daß beim menschlichen Insulinmangeldiabetes eine gleichartige Störung des Mesenchymstoffwechsels vorliegt. Unter dieser Voraussetzung wären die mitgeteilten Befunde geeignet, die Anhäufung von Grundsubstanz in den Organen des Diabetikers und insbesondere die Manifestation von Gefäßschäden verständlich zu machen.

The rate of synthesis and turnover of sulphated mucopolysaccharides was measured in alloxan-diabetic rats and in healthy controls with the aid of ${ }^{35} \mathrm{~S}$-sulphate.

In agreement with earlier findings, the alloxan-diabetic animals showed a decrease in the rate of synthesis of the sulphated mucopolysaccharides to about half that of the untreated controls. In the alloxan-diabetic animals, the biological half life of the sulphated mucopolysaccharides in various organs was increased to more than three times that in the control animals. The increase in the half life of the sulphated mucopolysaccharides therefore exceeded the inhibition of synthesis in the alloxan-diabetic animals.

Since the change in the turnover of SMPS ${ }^{1}$ ) described here is probably the direct result of the insulin deficiency, there is probably a similar disturbance in the mesenchymal metabolism in human insulin-deficiency diabetes. On the basis of this assumption, the present work would explain the accumulation of ground substance in the organs of diabetics, and especially the manifestation of blood vessel damage.

Seitdem durch die Insulintherapie extreme Stoffwechselentgleisungen bei Diabetes mellitus vermieden werden können und nur noch etwa $1,5 \%$ aller Zuckerkranken im Coma diabeticum sterben, sind in der Klinik die durch diese Stoffwechselerkrankung hervorgerufenen Komplikationen am Gefäßsystem zum bedeutsamen therapeutischen Problem geworden. Der diabetische Gefäßschaden betrifft sowohl das Kapillarsystem (vor allem in Form der diabetischen Retinopathie und der Glomerulosklerose) als auch die kleinen und großen Arterien.

Die kapillären, arteriolären und arteriellen Gefäßerkrankungen können unter dem Begriff der „diabetischen Angiopathie" zusammengefaßt werden. Sie stellt nach BürGER, HETENYI, RANDERATH und DIETZEL . (1-5) eine Gefäßerkrankung eigener Prägung dar. Art und Ausmaß der diabetischen Angiopathie sind von zahlreichen Autoren in den verschiedenen Organen untersucht worden (6-10).

Für die Deutung der Pathogenese diabetischer Spätkomplikationen gewinnen elektronenoptische Befunde an Kapillaren Bedeutung, die gezeigt haben, daß es in den Transitstrecken (Hauss und Mitarbeiter (11-14)) frühzeitig zu Ablagerungen von Grundsubstanz kommt und $\mathrm{daB}$ bei diabetischer Arteriosklerose die Transit- strecken durch diese Substanz dicht ausgefüllt sind (15). Gleichartige Veränderungen haben wir auch bei juvenilen Diabetikern nach mehrjähriger Krankheitsdauer beschrieben (Otro, THEMANN, WagNer (16)). Durch histochemische Untersuchungen konnten in den abgelagerten Substanzen vor allem Mukopolysaccharide und Glykoproteide, also Bestandteile der Grundsubstanz, sichergestellt werden.

In früheren Untersuchungen über den Stoffwechsel der sulfatierten Mukopolysaccharide bei alloxandiabetischen Ratten (17) konnten wir in Übereinstimmung mit den Untersuchungen von SCHILLER und DORFMAN $(18,19)$ sowie GlaubitT (20) einen verminderten ${ }^{35} \mathrm{~S}-S u l f a t e i n b a u$ in die sulfatierten Mukopolysaccharide der Gefäßwand feststellen. Zwischen diesem Befund, der auf eine verminderte Synthese von Grundsubstanz bei Alloxandiabetes hinwies, und der histochemisch nachweisbaren Vermehrung von Mukopolysacchariden und Glykoproteinen im diabetischen Bindegewebe -schien eine Diskrepanz zu bestehen, die bislang nicht geklärt wurde.

Die Frage, warum es bei verminderter Syntheseleistung trotzdem zu einem vermehrten Gehalt an Mukopoly-

1) Abkiirzung: SMPS = sulfatierte Mukopolysaccharide. 
sacchariden in der Gefäßwand kommt, haben wir jetzt durch zusätzliche Bestimmung der biologischen Umsatzraten bzw. der Geschwindigkeit des Abbaus der Mukopolysaccharide bei alloxandiabetischen Tieren geklärt. Nachfolgend werden die Befunde, die wir über den ${ }^{35}$ S-Sulfateinbau in die SMPS und über die SMPS-Halbwertszeit im Bindegewebe alloxandiabetischer Ratten exhoben haben, mitgeteilt.

\section{Versuchsanordnung und angewandte Methoden}

Die Untersuchungen wurden an männlichen Wistar-Ratten mit einem durchschnittlichen Körpergewicht von $150 \mathrm{~g}$ durchgeführt. Während der Versuche wurden die Tiere unter konstanten Bedingungen von Milieu und Fütterung gehalten.

Zur Erzeugung eines Alloxandiabetes erhielten 270 Tiere je $200 \mathrm{mg} / \mathrm{kg}$ Alloxan in frisch bereiteter 5proz. Lösung subkutan injiziert. Für die Versuche wurden diejenigen 72 Tiere ausgewählt, bei denen der Alloxandiabetes - nach dem Ergebnis mehrfacher Blutzucker- (enzymatisch), Harnzucker- und Harnacetonuntersuchungen - am stärksten ausgeprägt war. Der Diabetes blieb während der gesamten Versuchsdauer unbehandelt. Die Blutzuckerwerte lagen bei den alloxandiabetischen Ratten am Tag der ${ }^{35}$ S-Injektion zwischen 270 und $712 \mathrm{mg} / 100 \mathrm{ml}$.

3 Wochen nach Beginn des Alloxandiabetes wurde allen Tieren $1 \mathrm{mC}{ }^{35}$ S-Sulfat (Natriumsulfat, steril, trägerfrei in $0,5 \mathrm{~m} l$ physiol. $\mathrm{NaCl}-$ Lösung) intraperitoneal injiziert.

$\mathrm{Zu}$ bestimmten Zeiten nach ${ }^{35} \mathrm{~S}$-Sulfatinjektion, und zwar nach $12,24,48,96,192$ und 288 Stdn., wurden jeweils 12 Versuchstiere mit Alloxandiabetes und 12 Kontrolltiere getötet. Den Tieren wurden Herz, Aorta, Niere und Haut präparativ entnommen. Die Untersuchungen über den Stoffwechsel der sulfatierten Mukopolysaccharide mittels ${ }^{35}$ S-Sulfat wurden nach den früher bereits publizierten Methoden (17) durchgeführt. Es wurde die spezifische Aktivität der sulfatierten Mukopolysaccharide im Bindegewebe ( ${ }^{35} \mathrm{~S}-I m p . / M i n . / 100 \mu \mathrm{g}$ SMPS) ermittelt. Die Syntheseleistung von Mesenchymzellen ist an der Höhe der Inkorporationsraten von ${ }^{35} \mathrm{~S}$-Sulfat in die sulfatierten Mukopolysaccharide (SMPS) meßbar.

Die biologischen Umsatzraten der mit ${ }^{35} \mathrm{~S}$-markierten sulfatierten Mukopolysaccharide lassen sich durch Messung der Abnahme der spezifischen Aktivität der SMPS innerhalb eines bestimmten Zeitraumes erfassen. Als biologische Halbwertszeit der sulfatierten Mukopolysaccharide ist die Zeit definiert, in der die beobachtete höchste spezifische ${ }^{35}$ S-Aktivität der SMPS auf die Hälfte abgesunken ist.

\section{Ergebnisse}

Die Meßergebnisse der ${ }^{35}$ S-Sulfateinbauraten in die SMPS des Bindegewebes von Haut, Aorta, Niere und Herz sind in Abbildung 1 wiedergegeben. Es ist ersichtlich, daß die ${ }^{35}$ S-Sulfateinbauraten sowohl bei den normalen als auch bei den alloxandiabetischen Tieren in den einzelnen Organen unterschiedlich hoch liegen, worauf wir bereits vor Jahren aufmerksam gemacht haben. Stets liegen jedoch die Einbauraten 12 bzw. 24 Stdn. nach ${ }^{35}$ S-Sulfatinjektion bei den alloxandiabetischen Tieren niedriger als bei den Kontrolltieren, und zwar durchschnittlich etwa um 50\%.

Aus dem 12- bzw. 24-Stundenwert kann vornehmlich auf die Syntheseleistung der Mesenchymzellen geschlossen werden. Sie liegt demnach bei alloxandiabetischen Tieren eindeutig niedriger als bei den normalen.

Betrachten wir nun den Abfall der spezifischen Aktivität in den Organen, wie er sich aus den in Abbildung 1

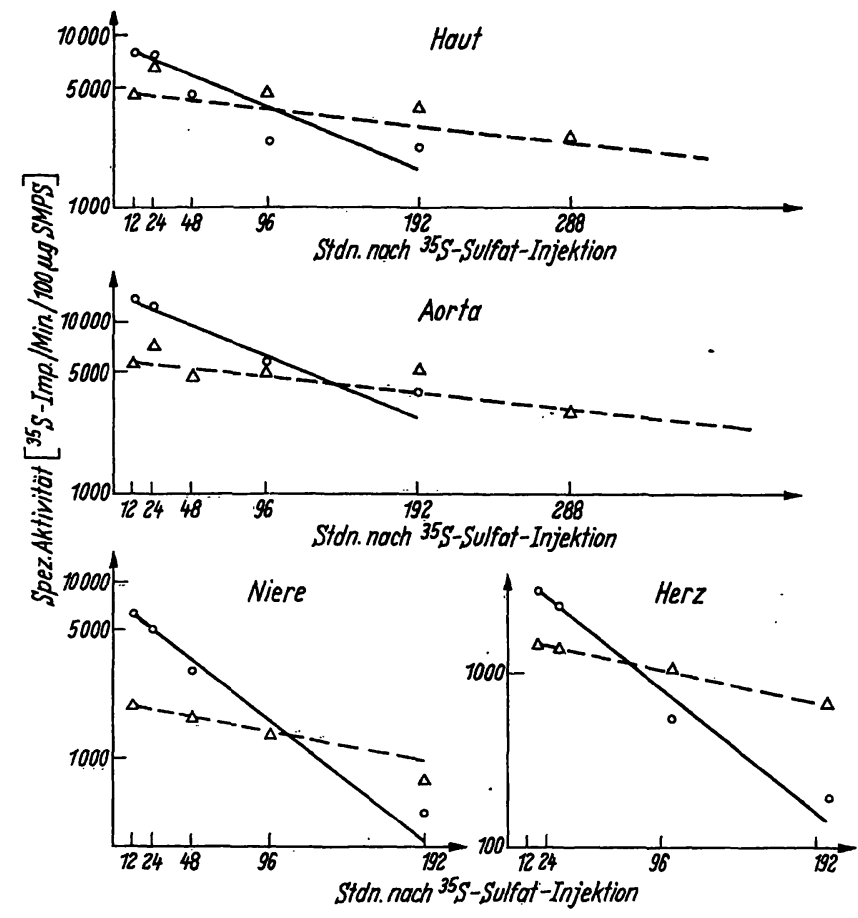

Abb. 1

Einfluß von Alloxandiabetes auf die SMPS-Synthese, gemessen als 35-S-Sulfat-Einbaurate, und auf die Abnahme der spezifischen ${ }^{35 S}$ Aktivität in den SMPS, gewonnen aus dem Bindegewebe ver$\Delta-\Delta=$ Alloxan-Diabetes, $\mathrm{O}^{-} \mathrm{o}=$ Kontrolltiere

eingezeichneten Meßwerten der spezifischen Aktivität bis zu 192 bzw. 288 Stdn. p. i. ergibt:

Wie wir bereits früher mitteilten, fallen bei den Normaltieren die spezifischen Aktivitäten in den Organen unterschiedlich schnell $a b$, in Haut und Aorta relativ langsam, in Niere und Herz schneller. Die aus diesen Daten ermittelten Halbwertszeiten der SMPS in den

Tab. 1

${ }^{35}$ S-Sulfat-Einbaurate und SMPS-Halbwertszeiten in Bindegewebe, das aus verschiedenen Organen normaler und alloxandiabetischer Ratten gewonnen wurde

\begin{tabular}{|c|c|c|c|c|}
\hline Organ & $\begin{array}{l}\text { SMPS-Syn- } \\
\text { these (Sppez- } \\
\text { Aktivität in } \\
\text { s5S-Imp/ } \\
100 \mu g \text { SMPS, } \\
12 \text { Stdn. nach } \\
\text { 25S-Sulfatin- } \\
\text { jektion }\end{array}$ & $\begin{array}{c}\text { Synthese- } \\
\text { Hemmung } \\
\text { (in \% der } \\
\text { Kontroll- } \\
\text { tiere) }\end{array}$ & $\begin{array}{c}\text { SMPS-Halb- } \\
\text { wertszeit in } \\
\text { Tagen }\end{array}$ & $\begin{array}{l}\text { HWZ-Ver- } \\
\text { längerung } \\
\text { (in \% der } \\
\text { Kontroll- } \\
\text { tiere) }\end{array}$ \\
\hline $\begin{array}{c}\text { Herz } \\
\text { Kontrolle }\end{array}$ & 3000 & & 2,25 & \\
\hline $\begin{array}{c}\text { Herz } \\
\text { Diabetes }\end{array}$ & 1515 & $\sim 50 \%$ & 7,25 & $322 \%$ \\
\hline$\underset{\text { Kontrolle }}{\text { Aorta }}$ & 13542 & 1 & 4,1 & \\
\hline $\begin{array}{c}\text { Aorta } \\
\text { Diabetes }\end{array}$ & 5906 & $\sim 56 \%$ & 13,35 & $326 \%$ \\
\hline$\underset{\text { Kontrolle }}{\text { Haut }}$ & 7982 & 1 & 4 & $\downarrow$ \\
\hline$\underset{\substack{\text { Haut } \\
\text { Diábetes }}}{c}$ & 4676 & $\sim 41 \%$ & 13 & $.325 \%$ \\
\hline $\begin{array}{c}\text { Niere } \\
\text { Kontrolle }\end{array}$ & 6170 & & 2,28 & $\downarrow$ \\
\hline $\begin{array}{c}\text { Niere } \\
\text { Diabetes }\end{array}$ & 2204 & $\sim 64 \%$ & 7,27 & $318 \%$ \\
\hline
\end{tabular}


verschiedenen Organen der Kontrolltiere sind in Tabelle 1 zusammengestellt und entsprechen den früher von uns publizierten Werten (17).

Bei alloxandiabetischen Ratten verläuft der Abfall der spezifischen Aktivität stets, von einem gegenüber den Kontrolltieren niedrigeren Ausgangsniveau ausgehend, ebenfalls in den verschiedenen Organen unterschiedlich schnell, am langsamsten in Haut und Aorta, schneller in Herz und Nieren. Zudem ist der Unterschied in der Abnahme der spezifischen Aktivität zwischen den normalen und den alloxandiabetischen Tieren deutlich: Abbildung 1 läßt erkennen, daß in allen Organen die Einbaurate bei den alloxandiabetischen Tieren nach 12 Stdn. niedriger liegt, da $B$ aber die spezifische Aktivität wesentlich langsamer abklingt, so $\mathrm{da} B$ in allen Organen spätestens nach $100 \mathrm{Stdn}$. der gemessene Wert bei den alloxandiabetischen Tieren höher liegt. Der Markierungsindex verschiebt sich also im Lauf der Tage. Die aus der Abnahme der spezifischen Aktivität errechneten Halbwertzeiten der SMPS, die in Tabelle 1 zusammen mit den Einbauraten für die verschiedenen Gewebe zusammengestellt sind, zeigen, da $\beta$ der Abbau der SMPS bei den alloxandiabetischen Ratten sehr viel langsamer erfolgt. Der Substratdurchsatz bedarf der etwa dreifach längeren Zeit oder, anders ausgedrückt, ein einmal eingebautes Molekül bleibt dreimal so lange im Gewebe liegen.

\section{Diskussion}

Unsere Untersuchungen haben zunächst noch einmal bestätigt, daß bei alloxandiabetischen Ratten die ${ }^{35}$ SSulfateinbaurate in die SMPS der Grundsubstanz aller Organe nach $12 \mathrm{Stdn}$. deutlich kleiner ist als bei gesunden Kontrolltieren. Es kann angenommen werden, $\mathrm{da} \beta$ es sich hierbei um eine Auswirkung des Insulinmangels handelt, da die Veränderung durch Insulintherapie bei alloxandiabetischen Ratten rückgängig gemacht werden kann (17). In Übereinstimmung damit steht auch, daß Insulin bei gesunden Tieren den ${ }^{35} \mathrm{~S}-$ Sulfateinbau beschleunigt.

Die Versuche zeigen des weiteren, daß die Halbwertszeit der aus den Organen alloxandiabetischer Tiere gewonnenen SMPS wesentlich länger ist als die der SMPS gesunder Tiere. Der Substratdurchsatz erfolgt demnach im Mesenchym alloxandiabetischer Ratten verzögert. Das Ausmaß der Verzögerung des SMPSAbbaus übertrifft das Ausma $\beta$ der Verminderung der SMPS-Synthese. Mit anderen Worten: Im alloxandiabetischen Organismus ist der Abbau der Grundsubstanz noch stärker verzögert als ihr Aufbau, so daß eine Anhäufung von Grundsubstanz die zwangsläufige Folge ist.

Nach den bislang vorliegenden Befunden muß angenommen werden, daß die beschriebenen Veränderungen des SMPS-Umsatzes direkte Folge des (alloxan-) diabetischen Insulinmangels sind. Insofern mag ein vorsichtiger Rückschluß auf die Verhältnisse beim Insulinmangeldiabetes des Menschen erlaubt sein. Die mitgeteilten Befunde würden verständlich machen, warum es beim Zuckerkranken zur Einlagerung von Grundsubstanz in das Mesenchym der Organe und zur Manifestation von Gefäßveränderungen, insbesondere auch an den Kapillaren (Nierenglomerula), kommt.

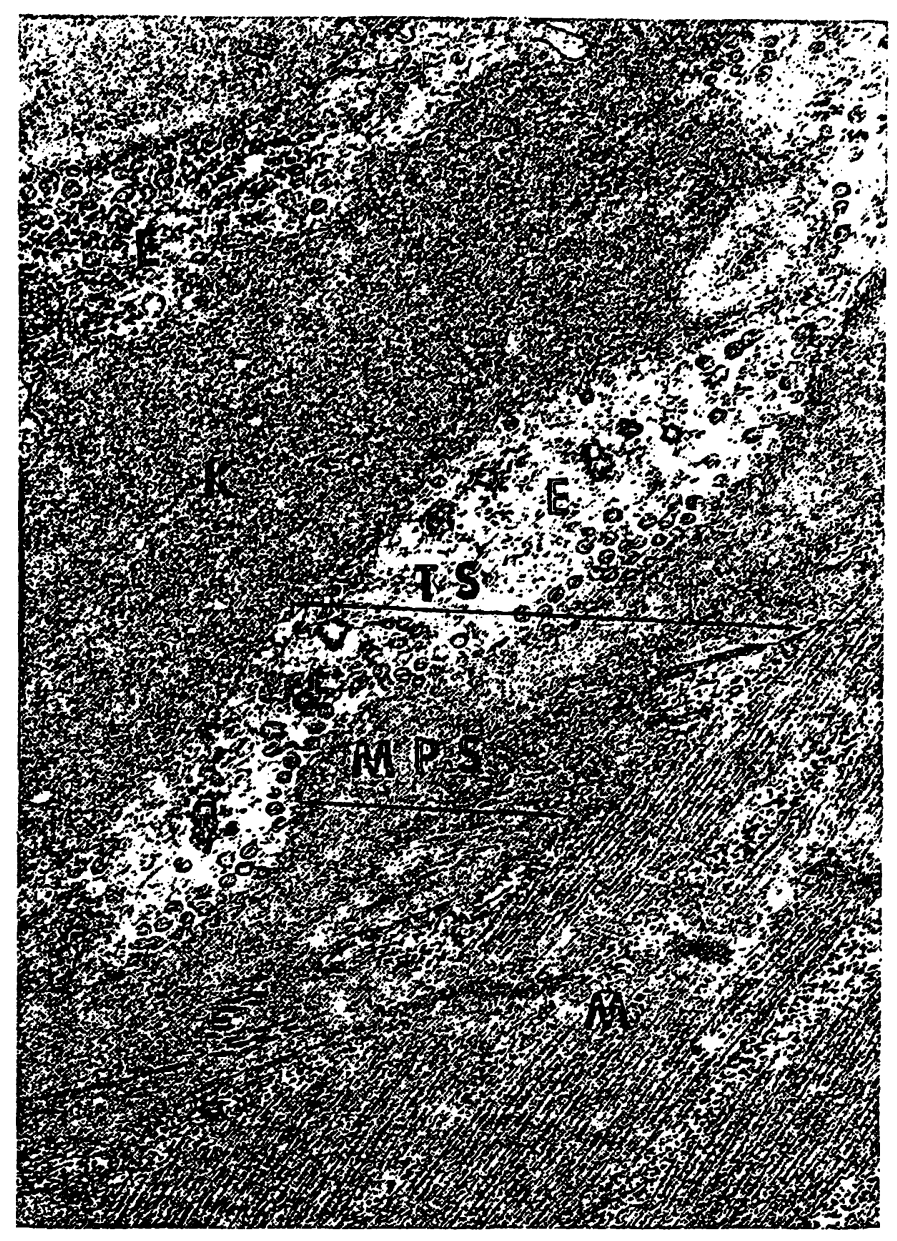

Abb. 2

Elektronenoptisches Bild einer Muskelexcision vom Zuckerkranken. Endothelzelle (E), Kapillarlumen (K), Transitstrecke (TS) und Anschnitt von Endothelzelle $(E)$ und Skelettmuskelzelle $(M)$ bei einem Patienten mit Diabetes mellitus. Charakteristisch ist die Substanzvermehrung in der MPS-Zone (MPS). Vergr.: 48000:1

Wir haben kürzlich in elektronenoptischen Bildern an Muskelexcisionen von Zuckerkranken gezeigt, daß in der Transitstrecke eine erhebliche Ansammlung von Bindegewebsgrundsubstanz besteht (Abb. 2) (15). Auf die Folgen, die diese vermehrte Einlagerung von Grundsubstanz in die Transitstrecke hat, haben wir dabei hingewiesen. Es kann zu einer Störung des Stofftransportes zwischen Kapillarlumen und Parenchymzelle kommen, die Zellnekrosen verursacht. Diese Transportstörung ist neben der Einengung der Arterienlumen mitverantwortlich für den Gliedmaßenbrand und die Herzmuskelnekrosen, die so häufig beim Zuckerkranken auftreten. In Fällen mit nachweislich gut durchgängigen Arterien ist sie sogar als einzige Ursache der Zellnekrose zu bewerten.

Der Deutschen Forschungsgemeinschaft und der LVA Westfalen sei für die Unterstützung bei der Durchführung dieser Untersuchiungen bestens gedankt. 


\title{
Literatur
}

1. Bürger, M., Angiopathia diabetica, Georg Thieme. Verlag, Stuttgart (1954). 2. BürGER, M., Altern und Krankheit, VEB Georg Thieme Verlag, Leipzig (1957). - 3. BürGER, M. und H. KNOBLOCH, Münch. med. Wschr. 101, 309 (1959). - 4. HetenYr, G., Acta med. Acad. Sci. Hung. IV, 425 (1963). - Randerath, E. und P. B. Dierzer, III. Kongr. Intern. Diabetes Fed. 1958, S. 54, Georg Thieme Verlag, Stuttgart (1959). - Angervall, L., G. Dotevall und K. LehmanN, Acta med. Scand. 169, 339 (1961). - 7. BencosME, S. A., R. O. West, J. W. KerR und D. L. Wilson, Amer. J. Med. 40, 67 (1966). - 8. Burstern, R., H. T. Blumenthal und S. Soule, Amer. J. Obstetr. Gynec. 74, 96 (1967). - 9. Fuchs, U., Frankf. Zschr. Path. 73, 318 (1964). - 10. Moore, J. M. und I. D. O. Frew, Brit. Med. J. 2, 19 (1965). - 11. Hauss, W. H., Verh. Dtsch. Ges. inn. Med. 69, 554 (1963). - Hauss, W. H., Verh.
Dtsch. Ges. f. Kreislforsch., 30. Tgg., 153 (1964). - 13. Hauss, W. H. und G. JUNGE-HüLSING, Hochdruckforschung II, S. 125, Georg Thieme Verlag, Stuttgart (1965) Symposium Freiburg. 14. JungrHülsing, G., Die Therapiewoche 16, 485 (1966). - 15. Hauss, W. H., G. Junge-Hürsing und H. Themann, Med. Welt 19, 7 (1968). - 16. Otro, H., H. Themann und H. Wagner, Klin. Wschr. 45, 299 (1967). - 17. Hauss, W. H., G. JUNGE-HüLsING und H. Oтто, in: Diabetische Angiopathie S. 229, Akademie Verlag, Berlin (1964): - 18. Schiller, S. und A. Dorfman, Biochim. biophysica Acta (Amsterdam) 16, 304 (1955). - 19. Schiller, S. und A. Dorfman, J. biol. Chemistry 227, 625 (1957). - 20. Glaubitr, D., Verh. Dtsch. Ges. f. Verdauungs- u. Stoffwechselkrankh., 22. Tagg. Wiesbaden 1964. S. 349, S. Karger Verlag, Basel (1965)

\section{Wirkungssynergismus Chondroitin-4-sulfat-Protein abbauender Enzyme des Arteriengewebes}

\author{
Von E. Held, O. Hoefele, G. Reich, U. Stein, E. Werries und E. Buddecke \\ Aus den Pbysiologisch-Chemischen Instituten der Universitäten Tübingen und Münster
}

(Eingegangen am 5. Dezember 1967)

\section{Herrn Prof. Dr. Dr. Ernst Scbütte qum 60. Geburtstag gewidmet}

Im Arteriengewebe des Rindes (Aorta thoracica) sind Hyaluronatglykanohydrolase (EC 3.2.1.35), Chondroitinsulfat-sulfohydrolase (EC 3.1.6.4), N-Acetyl- $\beta$-D-glucosaminidase (EC 3.2.1.30), $\beta$-D-Glucuronidase (EC 3.2.1.31), Kathepsin D (EC 3.4.4.23) und eine saure Carboxypeptidase (EC 3.4.2...) in spezifischen Aktivitäten von 0,01-10 mU/mg Extraktprotein bzw. 0,71-9,4 mU/g Frischgewebe vorhanden und können aus Homogenaten des Frischgewebes 80-1200 fach angereichert werden.

Die Enzyme zeigen bei der Reinigung z. T. gleiches Verhalten und synergistische bzw. successive Wirkung auf Chondroitin-4-sulfatProtein oder dessen Spaltprodukte.

Hyaluronat-glykanohydrolase (Hyaluronidase) und Kathepsin D bauen Kohlenhydrat- bzw. Proteinkomponente des Chondroitin-4-sulfat-Proteins gleichzeitig und ohne gegenseitige Beeinträchtigung ihrer Wirkung ab. Substrat der Arterien-Chondroitin-4-sulfat-sulfohydrolase ist Chondroitin-4-sulfat. Die Abspaltung des Estersulfats durch die Sulfatase wird durch die gleichzeitige Anwesenheit und Wirkung der Hyaluronidase nicht beeinflußt.

Die von der proteolytischen Einwirkung von Kathepsin D auf Chondroitin-4-sulfat-Protein freigesetzten Chondroitin-4-sulfat-Peptide werden durch die saure Carboxypeptidase des Arteriengewebes unter Abspaltung der C-terminalen Aminosäure weiter abgebaut. Der Wirkungssynergismus von Kathepsin D und saurer Carboxypeptidase zeigt sich durch Festlegung ihrer Spaltorte an der oxydierten BKette des Rinderinsulins.

$\beta$-D-Glucuronidase und N-Acetyl- $\beta$-D-glucosaminidase der Aorta lassen sich trotz 1000 facher Reinigung nicht voneinander trennen. In Anwesenheit von $\mathrm{N}$-Acetyl- $\beta$-D-hexosaminidase wird die $\beta$-D-Glucuronidase unter Verschiebung des $\mathrm{pH}-\mathrm{Optimums}$ von $\mathrm{pH} 5,2$ auf $\mathrm{pH} 4,5 \mathrm{pH}$-abhängig aktiviert.

Die Kenntnis der Eigenschaften und Wirkungsweise der Chondroitinsulfat-Protein abbauenden Enzyme erlaubt Rückschlüsse auf den Mechanismus und Abbauweg des Chondroitinsulfats im Arteriengewebe.

Hyaluronate glycanohydrolase (EC 3.2.1.35), chondroitin sulphate sulphohydrolase (EC 3.1.6.4), N-acetyl- $\beta$-D-glucosaminidase (EC 3.2.1.30), $\beta$-D-glucuronidase (EC 3.2.1.31), cathepsin D (EC 3.4.4.23) and an acid carboxypeptidase (EC 3.4.2. ...), with specific activities of $0.01-10 \mathrm{mU} / \mathrm{mg}$ extract protein or $0.71-9.4 \mathrm{mU} / \mathrm{g}$ fresh tissue, are present in bovine arterial tissue (Aorta thoracica) and can be purified $80-1200$ fold from homogenised fresh tissue.

After purification, the enzymes show partly similar activity and synergistic or successive activity towards chondroitin-4-sulphate-protein or its cleavage products.

Hyaluronate glycanohydrolase (hyaluronidase) and cathepsin D simultaneously degrade the carbohydrate and protein components of chondroitin-4-sulphate respectively with no mutual impairment of activity. Chondroitin-4-sulphate is a substrate of arterial chondroitin-4 sulphate sulphohydrolase. Removal of the ester sulphate by the sulphatase is not affected by the presence and simultaneous activity of hyaluronidase.

The chondroitin-4-sulphate-peptides, released by the proteolytic action of cathepsin D on chondroitin-4-sulphate, are further degraded with removal of the C-terminal amino acid by the acid carboxypeptidase of arterial tissue. The mechanism of the synergistic action of cathepsin D and acid carboxypeptidase is shown by locating their sites of cleavage in the oxidised B-chain of bovine insulin.

Despite a 1000 fold purification from the aorta, $\beta$-D-glucuronidase and $N$-acetyl- $\beta$-D-glucosaminidase could not be separated from each other. In the presence of $\mathrm{N}$-Acetyl- $\beta$-D-hexosaminidase, the $\beta$-D-glucutonidase exhibits a $\mathrm{pH}$-dependent activation, 'with a shift of $\mathrm{pH}$ optimum from 5.2 to 4.5 .

From this information on the properties and mode of action of enzymes that degrade chondroitin-sulphate-protein, it is possible to discuss the mechanism and pathway of degradation of chondroitin sulphate in arterial tissue. 